\title{
Analysis Of Session Initiation Protocol With VoIP In Multimedia Conferencing System
}

\author{
Muhammad Adnan Kaim Khani ${ }^{1}$, Malook Rind ${ }^{1}$, Asif Ali Wagan ${ }^{1}$, Abdul Hafeez Shaikh ${ }^{1}$, Mansoor Hyder Depar ${ }^{2}$, Raheel \\ Sarwar $^{1}$,Abida Luhrani ${ }^{1}$, Humair Nawaz ${ }^{1}$, Zeeshan Ahmed ${ }^{1}$ \\ 1-Department of Computer Science, Sindh Madressatul Islam University, Karachi, Pakistan. \\ adnankk12@gmail.com \\ 2-Information Technology Centre, Sindh Agriculture University, TandoJam, Pakistan. \\ mansoor.hyder@sau.edu.pk
}

\begin{abstract}
Today various applications like Command line client $(\mathrm{OH}$ phone, SIP), Video conferencing server (MCU), SIP answering machine, gatekeeper and gateways, Gnome Meeting. Session Initiation Protocol (SIP) is a multimedia conferencing protocol standard by ITU-T. Open SIP project is the most comfortable and most economical approach to develop an open source implementation of the SIP protocol standard in the form of a library. This Open SIP Library is written in and based on Open source library (OPSlip). Open SIP library is used to develop various applications like Command line client (OH phone, Callen-SIP), Video conferencing server (MCU), SIP answering machine, gatekeeper and gateways, Gnome Meeting. In this project, the first SIP Protocol standard is implemented using the Open SIP Project on two endpoints without a gatekeeper. $\mathrm{OH}$, phone, Callen-SIP, and NetMeeting were used for calling purpose. The performance analysis of this SIP protocol is done by measuring different QoS parameters like network bandwidth, Delay, Jitter and Packet loss. An investigation of the suitable tools led to the use of Ethereal and me perf as packet analyzers. Pack-ETH was used as a load generator. From the output of packet analyzers relative delay, jitter and packet loss are calculated. The result showed that protocol efficiency is more sensitive to changes in Jitter.
\end{abstract}

Key words: Video conferencing server; session initiation protocol (SIP); open SIP library; performance analysis

\section{INTRODUCTION}

In the last decade, the digital world has developed immensely. This presents and provides the conjunction between telecommunication and data-communication System. Circuit-switched networks were widely used for voice traffic before the arrival of packet-based networks. These circuitswitched networks provide qualitative voice transmission but can be highly insufficient. The alternative approach is to use packet-based networks which are very efficient but with a compromise on voice quality. Voice over Internet Protocol (VoIP) and Internet Protocol (IP) means delivering the efficiency of the packet-switched network even though be the equal of the voice quality of circuit switched network. As the telecommunication market is rushing towards IP based communication, competition has immensely increased the need to provide QoS in VoIP. VoIP processing and handling are shown below in figure1.1.

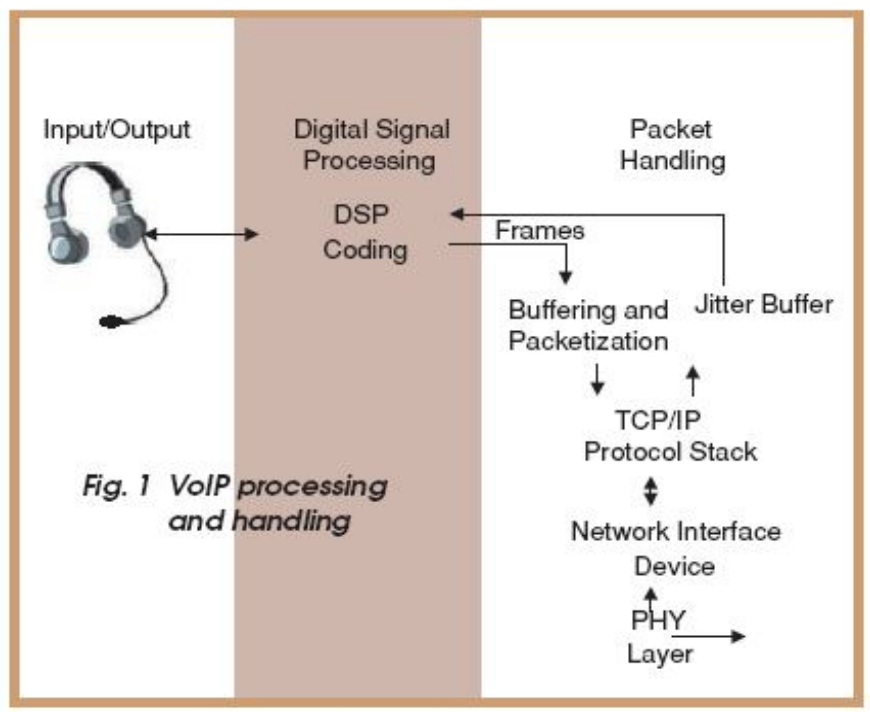

Fig. 1. VoIP processing and Handling

SIP Protocol Standard: It is a protocol presents multimedia information that includes voice, video, and facts. Therefore, a multimedia conferencing protocol is used primarily for packet-primarily based networks. Its variety includes registration admission reputation (RAS) manipulate, and contact setup was signaling as described in VoIP; call control as defined in sip; audio/video codecs (e.g., g.711 for audio and H.323 for video); and SIP real-time media transport protocols (RTP and RTCP). SIP is primarily based on reliable and conversation. 
Network Architecture: SIP network structure consists of terminals, gateways, and gatekeepers, multipoint control gadgets (multipoint controllers and multipoint processor). The range of SIP does now not contain the network interface, the human community, or the transport protocol used on the community. Instances of these networks include however aren't restricted to Fast Ethernet, Token Ring, Ethernet, FDDI, and ATM. The overall architecture of the system is defined in Fig. 2.

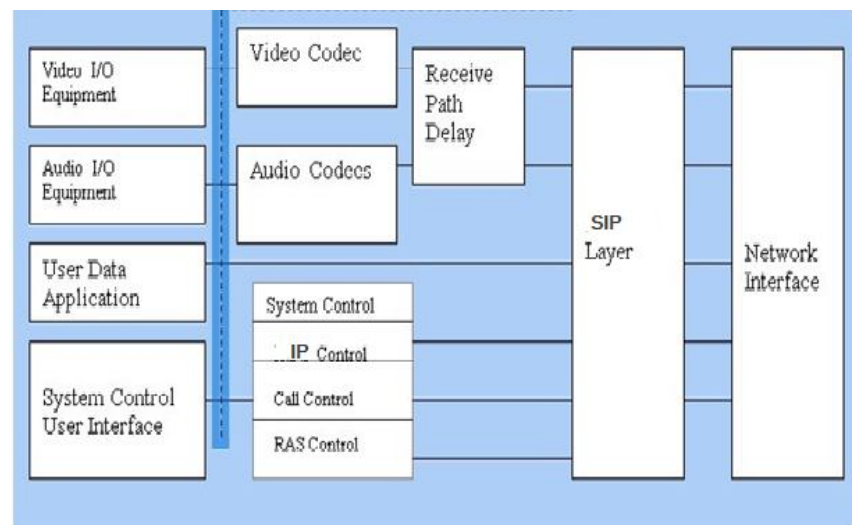

Fig. 2. Overall Architecture of the system

SIP terminals have features such as IP based and IP for signaling; RAS and RTP text, audio and video packets with G.711 codec for audio and video.

GATEWAYS: Interworking device is known as gateways. It is used to act as an interface between the various networks. A SIP gateway connects IP network to SCN networks (e.g., ISDN, PSTN) where an application of gateway is in IP telephony.

Characteristics: IP network flank the gateway of sip terminal and different can be MCU on SCN terminal. MCU is used as a terminal and left on the choice of producer. The gateway presents the necessary alteration. First of all, the gateway operates, however, inaddition to IP based signaling it starts to work as an MCU and has twin function for the same call.

In Pakistan, cybercrime and exploitation on the internet had remained a subject of extraordinary fear, however, needs mindfulness. The 0Strange blend of nature of assaults continually changing patterns of the exploitation, constrained information about immediate laws, which address cyber crimes in Pakistan and privileges of exploited people in instances of digital attacks,

\section{METHODOLOGY}

\section{A. SIP Call Signalling}

The Call signaling used to connect the endpoints by which the real-time data is to be transported. In two ways it can be done.

- Gatekeeper-Router call signaling: Call-signaling messages receive by Gatekeeper from one endpoint on signaling channel and route these messages to another endpoint.
- Direct Call-Signaling: During the admission confirmation the Gatekeeper communicates the endpoints that you can interchange messages straight.

\section{B. SIP Control Signaling:}

It used to interchange control messages between communicating endpoints. The SIP control messages are continuing on the SIP control channel which is a logical channel0, continuously open, unlike other channels. The control messages carried incorporate words to change the skills of terminals and to open and near logical channels.

\section{Capabilities Exchange}

It is the method via which speaking terminals exchange statistics concerning their transmitting and receiving abilities to the peer endpoint.

\section{Logical Channel Signaling:}

Itmilesuni-directional. It incorporates statistics from one endpoint to opportunity endpoint or multi-endpoints.

\section{KEY BENEFITS OF SIP}

\section{E. Independent of Network Architecture:}

As network technology evolves in the management of bandwidth and other things and SIP are used to enhance capabilities.

\section{F. Multipoint Support:}

Although without requiring a specialized multipoint control unit SIP can support conferences of three or more endpoints, for hosting multipoint conferences MCUs offer a more powerful and flexible architecture. In other components of a SIP system Multipoint capabilities can be included.

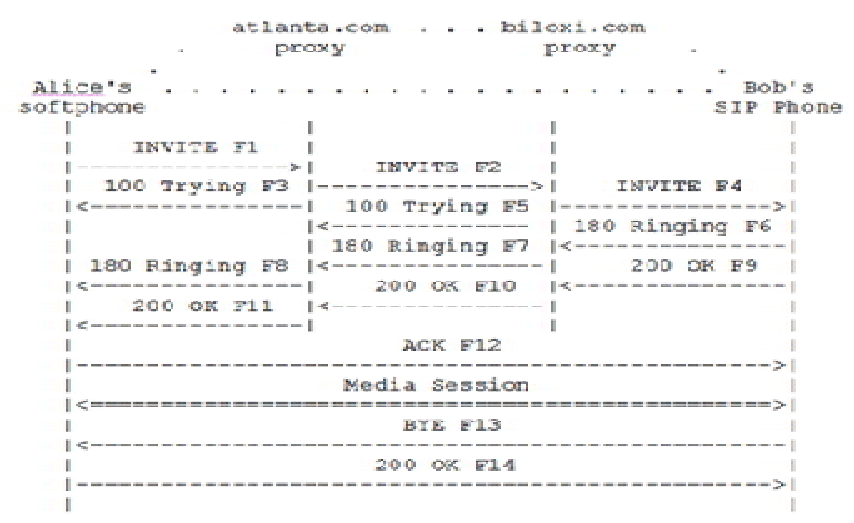

Fig. 3.Message Sequence

SIP is characterized in race controlled and confirmed by utilizing the net designing venture pressure (left) and depicts a typical taste call among the chatty weave and $\mathrm{x}$, containing a message accumulation (Figure 3 ).

Breaking this down, the call or consultation initiation desires to acquire several incredible capabilities, together with:

- $\quad$ Sign up a device for each consumer 
- $\quad$ Set up an information course between the two users

- $\quad$ Negotiate an information layout to use for voice facts

- $\quad$ Transmit voice records

\section{RESULTS}

Inside the exceptional broadcast communications condition, clients are irritating an ever-increasing number of refined media administrations and choices from their correspondence's backer organizations. For instance, making a telephone call from a pc connected to an IP network, and giving the determination over to a portable cell phone, signed into the wifi radio network after they need to leave the table. Or on the other hand, including video, conferencing or genuinely indeed changing records documents.

IP mixed media subsystem (IMS) characterizes an IP primarily based, bound together network structure that offers you this certificate of consistent versatility, providing customers with pervasive and adaptable inspire passage to such mixed media based unquestionably contributions as VoIP, video gushing, conferencing and records exchange, regardless of the stop gadget or get admission to course.

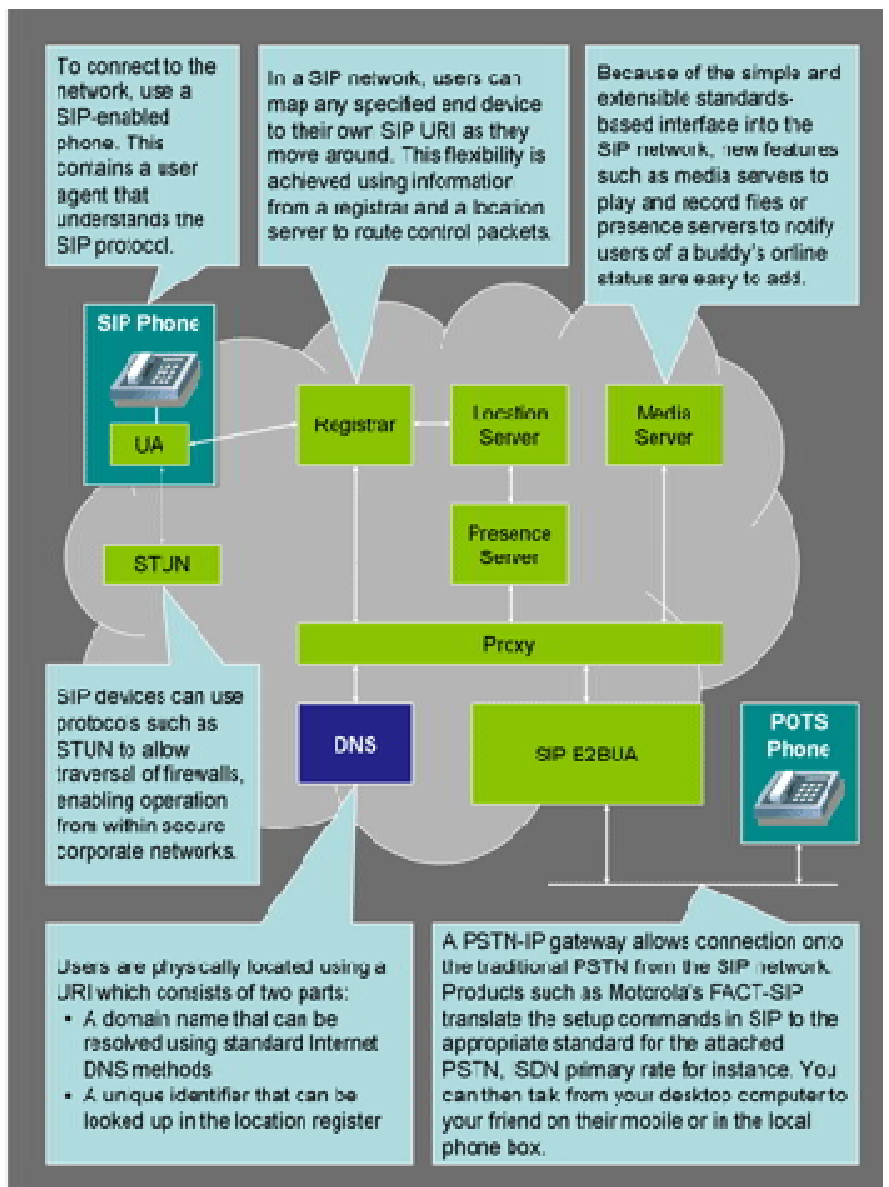

Fig. 4. Network Architecture
SIP is characterized in RFC controlled and confirmed by utilizing the net designing venture pressure (IETF), and depicts a typical taste call among the chatty weave and $\mathrm{x}$, containing a message accumulation (Fig. 4.).

\section{G. Registration}

As once in the past portrayed, while a client makes calls utilizing VOIP innovation, they require a record (like having a cellphone account or an email account). This offers them a totally one of a kind respected helpful asset identifier (URI) that enables you to make or achieve calls the character should friend a stop gadget, which fuses a pc or a taste empowered cellphone, with this URI. This technique is alluded to as enrollment.

\section{H. Initiate a call}

$\mathrm{X}$ includes a decision to call weave, so she enters bounces adapt to and starts a call, incurring X's UA to convey a taste welcome message to its decided-on intermediary. The endeavor for the intermediary is to gain the IP manage of bounce just so voice data might be steered between them that is alluded to like the taste revelation strategy.

This revelation is finished with the guide of the accompanying accumulation of exercises:

- the UA includes its one of a kind deliver to the welcome message (allowing message reactions to be steered bring down back close by a similar course).

- the intermediary at that point utilizes the space name server (DNS) to harvest the IP address of the intermediary in bounce's area.

- the changed welcome message is sent it straightforwardly to the recently settled IP manage (with the guide of default taste intermediaries focuses on port 5060).

Utilizing this methodology enables taste to use such blessing ability capacities in the network as simple content-based unquestionably identifiers and site guests fundamentally based load adjusting.

\section{Resolve the URI to IP address mapping}

As quickly because the invite message arrives at the appropriate proxy, it needs to be mapped to a particular IP deal with for y's sip cellular telephone.This is performed by the method for the utilization of looking into the URI inside the territory sign on. It will look at that point move again the present day IP address enlisted throughout stage 1 by the weave. Presently the welcome message can accordingly be sent to the surrender gadget. 


\section{J. Accept the call}

The accompanying mission to triumph over sooner than a name can be broad is to verify that the two closures of the decision are utilizing the equivalent records format. That is fathomed using records surpassed in some other convention alluded to as the conference portrayal convention (SDP). The taste welcome message from $\mathrm{x}$ also incorporates an SDP payload, which depicts the voice test value, pressure codec, and heaps of others, upheld with the guide of x's cellphone.

If weave's phone is pleasantly coordinated with the sort of configurations, sway is advised that $\mathrm{x}$ is endeavoring to name him (i.e., the cell phone will begin to ring). As fast as he arrangements the phone and acknowledges the decision, his IP manage is dispatched again to $\mathrm{x}$ in a response message, along the edge of a SDP payload containing the chose codec design, which at that point pursues a similar course came back to $\mathrm{x}$ because of the reality the welcome took at the way to weave. She will have the capacity to convey an acknowledgment message to flag then the setup is finished.

\section{K. Send voice data}

Presently every occasion has the breakthrough IP locations of the chance, and they're ready to start sending voice data among them. That is typically despatcher without a moment's delay shared (p2p) a large portion of the endpoints the use of over IP thus, it does never again venture through a similar way in light of the fact that the controllers over IP; as a result, it does no longer journey through the same path due to, manipulations. As fast because the session is introduced, the mission of taste is fundamentally whole. Examine that taste messages might be despatcher sometime of the voice call to supplant the setup, can deliver literary substance messages, or perhaps add different exercises to the call.

\section{Terminating the call}

As soon as $\mathrm{y}$ and $\mathrm{x}$ finish their communicate, setting up can regularly end the decision session. This reasons a sip bye message to be dispatched to the alternative birthday celebration within the call, that's then referred to. At this factor, the choice is terminated.

All the main goals have been achieved. Delay, packet lossand jitter were judged first for the no-load condition. For the Network, Iperf directly showed the increase in Packet loss, jitter, andDelay that can be seen comparing the output screenshots of Iperf under no load and load conditions. For SIP packets, light output was used. First SIP packets were filtered from the total traffic. Then additional column for the source was added for the source port. Then in display options, seconds since the previous frame was selected (seconds since the beginning of capture is the default option). Then continuous 15 packet stream was taken, and Delay was plotted against the Packet number.

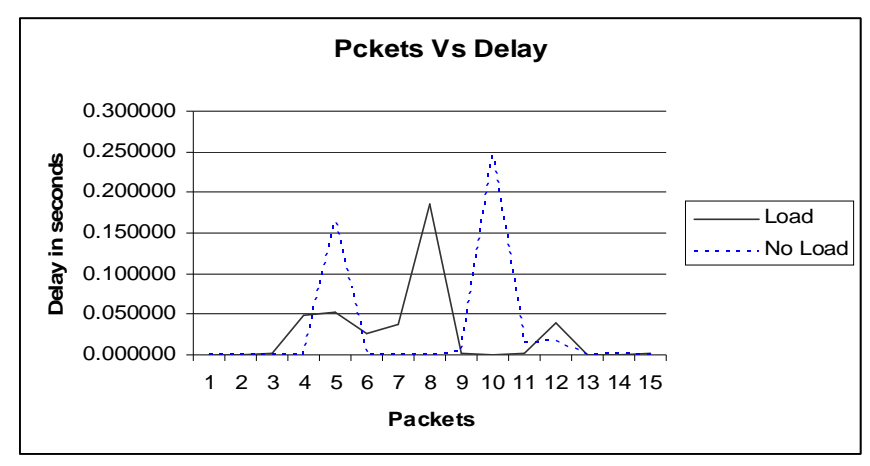

Fig. 4. Delay vs. packet number plot under loadand no-load.

Spikes in the graph are due to a single higher delay for logical channels or call proceedings, but overall it can be seen from figure 4 that packet delays and Jitter (variation in delay) under load conditions are much more than under no load conditions.

\section{CONCLUSION}

Results were in good theoretical range for all three metrics. Hence voice quality was excellent. Since the experiment was done on LAN involving just two endpoints, so even under high load delay and packet loss were in good-acceptable range. The only jitter was excessive which could be the single cause of decreased voice quality. One can say that the performance of SIP protocol is much sensitive to jitter than changes in delay \&loss. As can be unmistakable from the intermediary based structure and address lifecycle, taste is a smooth, green and particularly saleable answer this is meeting the developing name for VOIP correspondences - vast scale appropriation of the convention inside the venture grants for a lot of provider interoperability. In spite of the way that never again unequivocally treated in this pamphlet, taste network components additionally allow for future blast and the option of late skills by the method for the utilization of passing messages that incorporate unsupported fields straightforwardly through to the accompanying intermediary or stop man or lady.

Different vendors selling VOIP telephony equipment including CISCO use the dedicated network I/O cards with particular purpose DSP hardware running on the real-time OS. Hence if one wants to improve the performance than either device must be upgraded, or else code must be optimized. If code optimization is kept in mind, then there can be some areas where one could work. 


\section{REFERENCES}

[1] Mattila, Juhana. "Real-Time Transport Protocol." Department of Computer Science, University of Helsinki, Helsinki October 19 (2003).

[2] Liu, Hong, and Petros Mouchtaris. "Voice over IP signaling: H. 323 and beyond." IEEE Communications Magazine 38, no. 10 (2000): 142-148.

[3] Kamble R, Pateriya RK. Interoperability and Vulnerabilities in VoIP protocol (SIP, H. 323). Science. 2012 Jun;2(1).

[4] Talha SK, Barry BI. Evaluating the Impact of Encryption on Voice over Internet Protocol (VoIP) Systems. 2012.

[5] Murkute, P. V., \& Deshmukh, V. M. A Review of Implementation of VOIP Communication on Embedded system. IJCER(2015, 4(2), 46-49.

[6] Basicevic I, Popovic M, Kukolj D. Comparison of SIP and H. 323 Protocols. In2008 The Third International Conference on Digital Telecommunications (ICT 2008) 2008 Jun 29 (pp. 162-167). IEEE.
[7] Li J, Lei W, Si H. A comparison of multimedia conferencing frameworks. Journal of Networks. 2010 Jun $1 ; 5(6): 740$.

[8] Dagiuklas T, Galiotos P. Architecture and Design of an enhanced H. 323 VoIP Gateway. In2002 IEEE International Conference on Communications. Conference Proceedings. ICC 2002 (Cat. No. 02CH37333) 2002 (Vol. 2, pp. 1209-1213). IEEE.

[9] Toral-Cruz H, Torres-Roman D. Traffic analysis for IP telephony. 2nd International Conference on Electrical and Electronics Engineering 2005 Sep 7 (pp. 136-139). IEEE.

[10] Chang CY, Chen MS, Huang PH. An H. 323 gatekeeper prototype: design, implementation, and performance analysis. IEEE transactions on multimedia. 2004 Dec; 6(6):936-46.

[11] Sze HP, Liew SC, Lee JY, Yip DC. A multiplexing scheme for H. 323 voice-over-IP applications. IEEE Journal on Selected Areas in Communications. 2002 Sep;20(7):1360-8. 